\title{
HIGH-QUALITY ITERATIVE TOF MLEM RECONSTRUCTION FOR SHORT SCANS IN TOTAL-BODY J-PET
}

\author{
R.Y. Shopa* \\ National Centre for Nuclear Research, Otwock-Świerk, Poland
}

\begin{abstract}
We introduce a list-mode time-of-flight maximum likelihood expectation maximisation (TOF MLEM) image reconstruction algorithm for the total-body Jagiellonian PET (J-PET) scanner, using an analytical model for the system response matrix (SRM), estimated as a log-polynomial fit of the Monte Carlo simulated emissions of backto-back $\gamma$-photons for each bin. Using the GATE software, the updated method is tested on the simulated NEMA IEC phantom, scanned over a short 35-second time by the 14O-cm long 24-module J-PET. By comparison to the reference TOF MLEM without the resolution modelling for the detectors from the CASToR framework, a significant improvement in image quality was observed. The inclusion of the penalisation into the reconstruction algorithm may achieve outcomes comparable to 50o-second scans, with the best results obtained for the anisotropic median-diffusion with a finite-impulse-response median hybrid filter. The proposed TOF MLEM can also be extended to account for the non-collinearity, positron range and other factors.
\end{abstract}

Keywords: J-PET, total-body PET, medical imaging, maximum likelihood expectation maximization

\section{INTRODUCTION}

Positron emission tomography (PET) is a diagnostic method for monitoring the metabolic processes in tissues using radioactive tracers as the source of the positrons $[1,2]$. The recent development of the highly sensitive total body (TB) PET scanners allows to perform molecular imaging of the whole human body with significant reduction of times of scan [3-6]. However, as these systems utilise hundreds of thousands LYSO crystal detectors, their cost could exceed $\$ 10 \mathrm{mln}$ [6]. The novel Jagiellonian PET (J-PET) scanners offer the alternative, using Compton scattering of the positron-electron annihilation photons in plastic scintillators for their detection, with the information of time-of-flight (TOF) also available $[4,7-12]$. A recent study, conducted for the simulated modular TB J-PET prototype, designed as a multi-layer scanner to compensate for lower detection efficiency, revealed its characteristics to be potentially superior to the clinical PET systems [13].

As a reliable image reconstruction algorithm, the maximum likelihood expectation maximization method (MLEM) is widely approved for PET imaging [14]. It utilises a probabilistic linear model, defined as a system response matrix (SRM), between the image and projection spaces of the detected events. SRM is usually decomposed into several matrices which account for geometric projections, normalisation, attenuation or resolution effects [15]. However, the complex detector geometry such as in modular TB J-PET, while strongly affecting SRM, is almost impossible to estimate.
In this work, we derive an SRM model for the modified TOF MLEM as a set of polynomial fits of the Monte Carlo simulated detector response to the emissions of back-to-back $\gamma$-photons that cover the field-of-view (FOV) of the TB J-PET scanner. Using the GATE software [16], the algorithm is tested on the simulated data for the IEC NEMA phantom, approved for the analysis of image quality, in a 140-cm long modular TB J-PET [17]. We validate TOF MLEM for the short scan, with and without penalisation, and compared with the reference algorithm from the CASToR framework that does not account for the detector blurring effects [18].

\section{METHODS}

\subsection{List-mode MLEM}

The MLEM algorithm is an iterative method of finding the highest conditional probability $P(\hat{\mathbf{y}} \mid \lambda)$ that the detected emission from the voxel $\lambda$ is measured as a response $\hat{\mathbf{y}}$ from the detector, assuming the PET data undergoes Poisson distribution [14]. That substantiates a set of linear equations:

$$
\left\langle y_{i}\right\rangle=\sum_{j \in J} m_{i j} \lambda_{j}
$$

where $\left\langle y_{i}\right\rangle$ is a forward projection - an expected number of the detected emissions by the $i$-th detector pair (bin), $\lambda_{j}$ is the intensity of the $j$-th voxel and $m_{i j}$

\footnotetext{
* Roman.Shopa@ncbj.gov.pl
} 
is a SRM element - the probability that an event coming from $\lambda_{j}$ is detected by the $i$-th bin. To avoid splitting the measured projection data $\hat{\mathbf{y}}$ into bins, a listmode MLEM is used [19], when the summation is made over each detected emission $e \in E$, resulting in the iterative formula to update $\lambda_{j}$ after the $n$-th iteration:

$$
\lambda_{j}^{(n+1)}=\frac{\lambda_{j}^{(n)}}{\sum_{i \in I} A_{i} N_{i} m_{i j}} \sum_{e \in E} \frac{m_{i_{e} j}}{\sum_{k \in J} m_{i_{e} k} \lambda_{k}^{(n)}}
$$

where $A_{i}$ and $N_{i}$ are the attenuation and (unrelated to SRM) normalisation factors, respectively.

The complex J-PET geometry requires a proper resolution modelling (RM), but the geometrical and the blurring factors of SRM are difficult to calculate analytically. An alternative solution was proposed in Ref. [20], reducing the bin space to $I \subset \Pi^{2}:$ a $2 \mathrm{D}$ Monte Carlo simulation is conducted for the emissions from pixels on the transverse plane only, which gives the unconditional probabilities $m_{i j}$ for the obliqueness angle $\theta=0$. The J-PET detectors are continuous in axial $(Z)$ direction (Figure 1, a), hence such SRM can be approved for a relatively small $(<50 \mathrm{~cm})$ axial FOV.

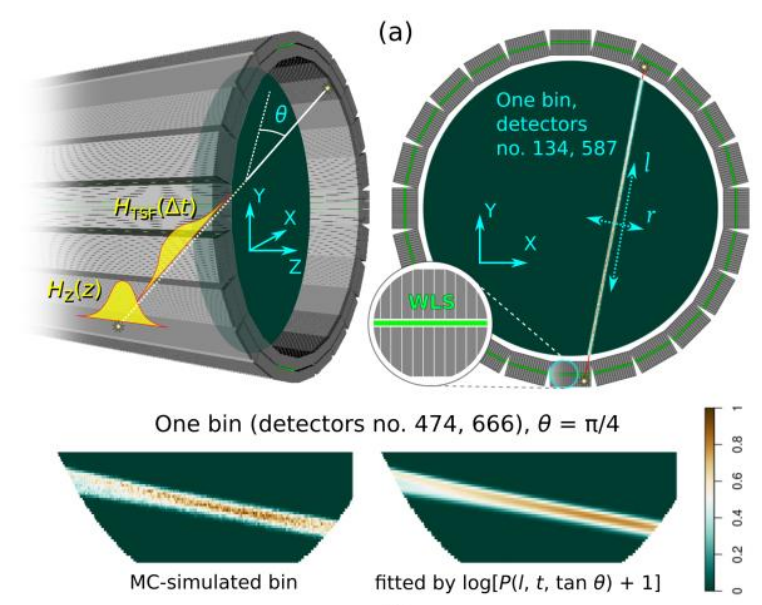

(b)

Figure 1. Schematic depiction of the bin variables $l, r$ and $\theta$ in the 2-layer modular J-PET (a); simulated and fitted detection probability distribution for an exemplary bin (b). WLS strips are shown in green, TSF and axial kernels $H_{\mathrm{TSF}}(\cdot)$ and $H_{Z}(\cdot)-$ visualised in yellow (scales are exaggerated)

We modified the approach by performing a set of five $2 \mathrm{D}$ simulations, $1 \mathrm{mln}$ per pixel, adjusting the obliqueness in range $\theta \in[0, \pi / 4]$. The first scatter in scintillators along the path of a photon was treated as the detection event. Next, a fit by a $\log [P(\cdot)+1]$ function, where $P(\cdot)$ is a polynomial of the 5 -th order, was conducted for each bin, using $\tan \theta$ and the transverse variables $l$ and $r$ as the arguments (Figure 1). Eventually, SRM was represented as a set of coefficients for the unique log-polynomial functions $m_{i}(l, r, \tan \theta)$. The symmetry of the J-PET scanner allows using less bins, reducing the SRM size below $100 \mathrm{MB}$, as i covers only $1 / 8$-th of the bin space $I$ [20].
We accounted for TOF and the uncertainty of the scattering (hit) position measurement along $Z$ as proposed in Ref. [20]: SRM elements are multiplied by the additional Gaussian time-spread-function (TSF) and axial kernels $-H_{\mathrm{TSF}}(\cdot)$ and $H_{Z}(\cdot)$, respectively. That implies the following substitution in Eq. (2):

$$
m_{i_{e} j} \rightarrow m_{i_{e}}\left(l_{j}, r_{j}, \tan \theta_{e}\right) \cdot H_{\mathrm{TSF}}\left(\Delta t_{e}\right) \cdot H_{Z}\left(z_{j}\right),
$$

where $\Delta t_{e}=t_{1}^{(e)}-t_{2}^{(e)}$ is the difference between the times of hits and $z_{j}$ is the axial coordinate of the $j$-th voxel. Geometric visualisation of $H_{\mathrm{TSF}}(\cdot)$ and $H_{Z}(\cdot)$ is given in Figure 1, a.

The attenuation and normalisation in Eq. (2) require an additional summation over $\theta$ (see Ref. [18]) since $I$ covers transverse plane only:

$$
\sum_{i \in I} A_{i} N_{i} m_{i j} \rightarrow \sum_{i \in I} \int_{\theta} A_{i, \theta} N_{i} m_{i}\left(l_{j}, r_{j}, \tan \theta\right)
$$

\subsection{Penalisation}

Penalisation of MLEM is employed to compensate for the excessive noise propagation and a positive bias in the image, which is particularly relevant to low statistics of measured emissions [21]. In one-step-late algorithms, a penalisation term is introduced as the derivative of the energy function $U(\lambda)$, defined by the Gibbs probability distribution [22]. It transforms the update formula (2) as follows:

$$
\lambda_{j}^{(n+1)}=\frac{\lambda_{j}^{(n)}}{\sum_{i \in I} A_{i} N_{i} m_{i j}+\left.\beta \frac{\partial}{\partial \lambda_{i}} U(\lambda)\right|_{\lambda_{j}^{(n)}}} \sum_{e \in E} \frac{m_{i_{e} j}}{\sum_{k \in J} m_{i_{e} k} \lambda_{k}^{(n)}},
$$

where $\beta$ is a the Bayes weight of the prior.

The median root prior (MRP) utilises the median filter, applied to the prior iteration $M\left(\lambda_{j}^{(n)}\right)$ [23].

$$
\left.\frac{\partial}{\partial \lambda_{j}} U(\lambda)\right|_{\lambda_{j}^{(n)}}=\frac{\lambda_{j}^{(n)}-M\left(\lambda_{j}^{(n)}\right)}{M\left(\lambda_{j}^{(n)}\right)} .
$$

The maximum-a-posteriori (MAP) expectation maximisation defines a custom prior function $U(\lambda)$ to calculate the penalisation [22]. We will employ MAP with the relative difference penalty (RD-MAP) [24]:

$$
U(\lambda)=\sum_{j \in J} \sum_{k \in N_{j}} \frac{\left(\lambda_{j}-\lambda_{k}\right)^{2}}{\left(\lambda_{j}+\lambda_{k}\right)+\gamma\left|\lambda_{j}-\lambda_{k}\right|},
$$

where $N_{j}$ covers the neighbouring voxels to $\lambda_{j}$ and $\gamma$ controls the degree of edge-preservation.

Finally, an anisotropic median-diffusion (AMD) filter was tested [25]. After each MLEM iteration (2), the following update is performed:

$$
\lambda_{j}^{(n)}=\lambda_{j}^{(n)}+\frac{\omega}{\left|N_{j}\right|} \sum_{k \in N_{j}} g\left(\left|\nabla \lambda_{j, k}^{(n)}\right|\right) \nabla \lambda_{j, k}^{(n)} .
$$


Here, $\omega \in[0,1]$ is a rate of diffusion, the gradient $\nabla \lambda_{j, k}=\lambda_{k}-\lambda_{j}$ and the diffusion function depends on yet another parameter $K$ (see Ref. [25]):

$$
g(x)= \begin{cases}\frac{25}{16 K}\left[1-\left(\frac{x}{\sqrt{5} K}\right)^{2}\right]^{2}, & |x| \leq \sqrt{5} K \\ 0, & \text { otherwise }\end{cases}
$$

After having applied AMD, the image is filtered as $\lambda_{j}^{(n)}=M\left(\lambda_{j}^{(n)}\right)$, to remove large noise spikes. We utilise an alternative approach - the finite-impulse-response median hybrid (FMH) filter, less sensitive to the voxel size [26]. This method calculates the median for a set of 13 linear sub-filters, applied to the combinations of the central voxel and its two neighbours in all possible directions (see Ref. [26] for the details).

\subsection{Simulation setup}

The J-PET scanner, constituted by 24 modules surrounding a cylinder of the radius $R=383 \mathrm{~mm}$, was defined for the simulated experiment in the GATE framework [16]. Each module was composed of 32 elongated BC-408 scintillator strips of the size $6 \mathrm{~mm} \times$ $30 \mathrm{~mm} \times 1400 \mathrm{~mm}$, arranged in 2 layers (Figure 1, a), with silicon photomultipliers (SiPM) attached at each end for the detection of optical signals, produced as a result of Compton scattering [13]. To improve axial resolution for hits (scattering points in detectors), a layer of wavelength shifters (WLS) was put in the 3$\mathrm{mm}$ gap between the layers [27]. WLSs are shown in green in Figure 1, a.
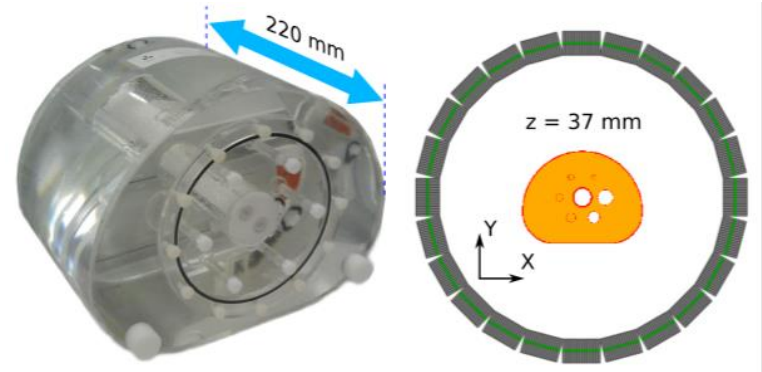

Figure 2. NEMA IEC phantom and its location in the TB JPET scanner. The colours on the right denote plastic (red) and water (orange)

The NEMA defined IEC phantom of the length 220 mm was simulated at the centre of the FOV (Figure 2), with four hot and two cold spheres of the phantom of the diameters $10 \mathrm{~mm}, 13 \mathrm{~mm}, 17 \mathrm{~mm}, 22 \mathrm{~mm}, 28 \mathrm{~mm}$ and $37 \mathrm{~mm}$, respectively [17]. The phantom was filled by radioactive water with the dissolved ${ }^{18} \mathrm{~F}$ FDG, with the fraction between the activities in the hot spheres and background volume was 4:1, amounting to $53 \mathrm{MBq}$ in total.

The simulation was launched to generate back-toback annihilation $\gamma$-photons of energy $511 \mathrm{keV}$, accounting for the photoelectric effect, Compton scattering, gamma conversion, Rayleigh scattering, ionisation and bremsstrahlung [28]. Total acquisition time was set to $500 \mathrm{~s}$. The data was then post-selected by a 3-ns time window, to get the coincident interactions - the times and positions of hits (see Ref. [28]). Only true events have been considered, ignoring random and scattered ones - both inside the phantom and the scintillators (ongoing studies are conducted on the latter issues - see e.g. Ref. [12]), amounting to $153 \mathrm{mln}$ in total. The assessed time and axial resolution CRT $=$ $191 \mathrm{ps}$ and $\mathrm{FWHM}_{\mathrm{Z}}=5 \mathrm{~mm}$, respectively, were used to post-smear the times and axial positions of hits [13]. Finally, a pre-defined NEMA IEC attenuation map (Figure 2, right) was used to calculate $A_{i, \theta}$ in Eq. (4) by the accelerated Siddon algorithm [29].

\section{RESULtS}

For most of the studies, we analysed a 10-mln subset of true evens, representing a 35 -second scan. Having assigned symmetric $2.5 \mathrm{~mm} \times 2.5 \mathrm{~mm} \times 2.5 \mathrm{~mm}$ size for the voxels $\lambda_{j}$, the list-mode TOF MLEM was launched for 20 iterations. The standard deviations of $H_{\mathrm{TSF}}(\cdot)$ and $H_{Z}(\cdot)$ kernels were set to reflect the $\mathrm{J}$-PET resolution CRT $=191 \mathrm{ps}$ and $\mathrm{FWHMz}=5 \mathrm{~mm}$.

In order to estimate the NEMA defined image quality parameters - contrast recovery coefficient (CRC) and background variability (BV) - twelve circular regions-of-interest (ROIs) were drawn on the transverse plane that intersect the spheres of IEC phantom, replicated at $z= \pm 10 \mathrm{~mm}$ and $z= \pm 20 \mathrm{~mm}$ (see Ref. [17] for the details). In addition, the root of mean squared error (RMSE) between $\lambda_{j}$ and known radiotracer distribution $\lambda_{j}^{\mathrm{GT}}$ (ground truth) was monitored:

$$
\mathrm{RMSE}=\sqrt{\frac{1}{N_{\text {vox }}} \sum_{j=1}^{N_{\text {vox }}}\left(\lambda_{j}-\lambda_{j}^{\mathrm{GT}}\right)^{2}},
$$

where $N_{\text {vox }}$ is the total number of voxels. For convenience, we restricted the volume to a $300 \mathrm{~mm} \times$ $240 \mathrm{~mm} \times 240 \mathrm{~mm}$ box around the phantom.
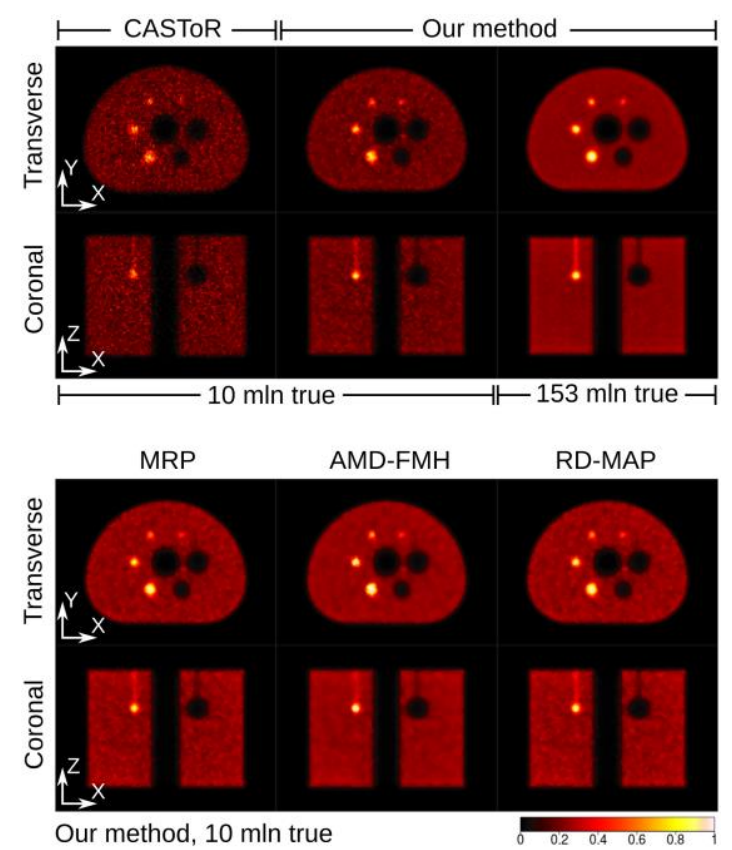

Figure 3. Transverse $(z=37 \mathrm{~mm})$ and axial $(y=0 \mathrm{~mm})$ crosssections of the NEMA IEC phantom, reconstructed by TOF MLEM (5-th iteration), depending on the algorithm version and the statistics of the measured events (top), or on the penalisation applied (bottom, see the details in the text) 
Figure 3, top depicts the cross-sections of the NEMA IEC phantom across the hot spheres, reconstructed by TOF MLEM from CASToR without RM and by our modified method. The images are shown for the 5th iteration, which exhibited the lowest RMSE both for the 10-mln and the full, 153-mln statistics (for $500 \mathrm{~s}$ ). Evidently, the realistic SRM improves the image quality and reduces the noise level.

The bottom images in Figure 3 reveal the results for our algorithm with penalties applied. We adjusted the regularisation parameters to achieve moderate effect with similar RMSE levels estimated for the 5 -th iteration: $\beta=0.06$ (MRP), $\omega=0.05, K=0.05$ (AMDFMH) and $\beta=0.03, \gamma=10.0$ (RD-MAP). A box-shaped $3 \mathrm{px} \times 3 \mathrm{px} \times 3 \mathrm{px}$ mask was utilised for the median filter of MRP. All variations clearly reduce noise, with AMD-FMH looking the most promising.
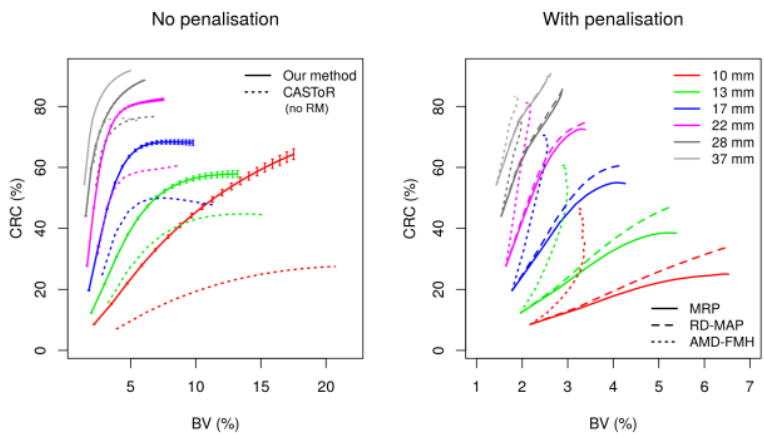

Figure 4. Image quality evolution over 20 TOF MLEM iterations for six spheres of the NEMA IEC phantom (10-mln true coincidences), depending on the version of the algorithm (left) or on the penalisation (right, see the details in the text).

Vertical ticks on the left mark the standard errors of CRC

Figure 4, left depicts CRC(BV) curves, estimated for a single reconstruction, averaged over 30 different choices of ROIs. Taking to account the standard errors for CRC denoted by vertical ticks for our TOF MLEM, we conclude that the proposed algorithm is superior to the one without RM from the CASToR framework.

Like in Figure 3, bottom, the AMD-FMH penalisation results in better image quality, as seen in Figure 4, right. This approach not only reduces BV to about $3 \%$ but preserves this level over iteration progress. The lowest RMSE is achieved at the $5^{\text {-th }}$ iteration and is comparable to the reconstruction made for the full 153-mln dataset.

Table 1 presents the CRC and BV levels for three spheres (two hot and one cold) of NEMA IEC, along with the corresponding RMSEs (using a normalized $[0 ; 1]$ scale), for all considered cases shown in Figure 3 . The numbers represent the 5 -th iteration and $10-\mathrm{mln}$ true events, except the final row (153 mln). As we see, the proper penalisation might improve the image obtained for a short scan, to a level comparable with a much longer, 500-second acquisition time.

\section{Discussion}

The introduced list-mode TOM MLEM capitalises on the unique geometry of the J-PET tomographs. Thanks to the continuous strips along $Z$-axis, it is possible to define SRM analytically for TB scanners without extending its size. It also reduces the number of the $2 \mathrm{D}$ simulations required for log-polynomial fitting, since the functions $m_{i}(l, r, \tan \theta)$ depend weakly on $\theta$. The simulations and the iterations can be launched in parallel, using GPGPU processors or advanced electronics, likewise for the simplified TOF MLEM in Ref. [20].

Table 1. Estimated RMSE and image quality parameters for the three spheres in the NEMA IEC phantom, reconstructed by the TOF MLEM at the 5-th iteration. Standard errors are given in parentheses

\begin{tabular}{|c|c|c|c|c|c|c|}
\hline \multicolumn{3}{|c|}{ CRC, \% } & \multicolumn{3}{|c|}{ BV, \% } & \multirow{2}{*}{$\begin{array}{l}\text { RMSE, } \\
\text { a.u. }\end{array}$} \\
\hline $13 \mathrm{~mm}$ & $22 \mathrm{~mm}$ & $28 \mathrm{~mm}$ & $13 \mathrm{~mm}$ & $22 \mathrm{~mm}$ & $28 \mathrm{~mm}$ & \\
\hline \multicolumn{7}{|c|}{ Reference (CASToR, no RM) } \\
\hline $\begin{array}{l}40.75 \\
(0.41)\end{array}$ & $\begin{array}{c}57.75 \\
(0.25)\end{array}$ & $\begin{array}{c}73.84 \\
(0.04) \\
\end{array}$ & $\begin{array}{c}8.57 \\
(0.62)\end{array}$ & $\begin{array}{c}5.03 \\
(0.25)\end{array}$ & $\begin{array}{c}4.08 \\
(0.21)\end{array}$ & 0.0845 \\
\hline \multicolumn{7}{|c|}{ No penalization } \\
\hline $\begin{array}{l}43.32 \\
(0.37) \\
\end{array}$ & $\begin{array}{r}73.58 \\
(0.15) \\
\end{array}$ & $\begin{array}{c}75.16 \\
(0.03) \\
\end{array}$ & $\begin{array}{c}5.55 \\
(0.34) \\
\end{array}$ & $\begin{array}{c}3.49 \\
(0.17) \\
\end{array}$ & $\begin{array}{c}2.95 \\
(0.12) \\
\end{array}$ & 0.0646 \\
\hline \multicolumn{7}{|c|}{$\operatorname{MRP}(\beta=0.06)$} \\
\hline $\begin{array}{l}34.47 \\
(0.24) \\
\end{array}$ & $\begin{array}{l}67.45 \\
(0.13) \\
\end{array}$ & $\begin{array}{l}73.53 \\
(0.03) \\
\end{array}$ & $\begin{array}{c}4.21 \\
(0.24) \\
\end{array}$ & $\begin{array}{c}2.81 \\
(0.11) \\
\end{array}$ & $\begin{array}{c}2.44 \\
(0.08) \\
\end{array}$ & 0.0430 \\
\hline \multicolumn{7}{|c|}{ RD-MAP $(\beta=0.03, \gamma=10.0)$} \\
\hline $\begin{array}{c}36.81 \\
(0.23) \\
\end{array}$ & $\begin{array}{l}67.65 \\
(0.12) \\
\end{array}$ & $\begin{array}{l}73.66 \\
(0.03) \\
\end{array}$ & $\begin{array}{c}4.04 \\
(0.22) \\
\end{array}$ & $\begin{array}{c}2.72 \\
(0.11) \\
\end{array}$ & $\begin{array}{c}2.40 \\
(0.08) \\
\end{array}$ & 0.0423 \\
\hline \multicolumn{7}{|c|}{$\operatorname{AMD}-F M H(\omega=0.05, K=0.05)$} \\
\hline $\begin{array}{l}39.99 \\
(0.16) \\
\end{array}$ & $\begin{array}{r}72.14 \\
(0.12) \\
\end{array}$ & $\begin{array}{l}70.73 \\
(0.03) \\
\end{array}$ & $\begin{array}{c}2.84 \\
(0.13) \\
\end{array}$ & $\begin{array}{c}2.12 \\
(0.06)\end{array}$ & $\begin{array}{c}1.94 \\
(0.05) \\
\end{array}$ & 0.0414 \\
\hline \multicolumn{7}{|c|}{ Full dataset (153 mln true, no penalisation) } \\
\hline $\begin{array}{l}43.77 \\
(0.10)\end{array}$ & $\begin{array}{c}71.55 \\
(0.08) \\
\end{array}$ & $\begin{array}{l}75.81 \\
(0.01) \\
\end{array}$ & $\begin{array}{c}1.86 \\
(0.08)\end{array}$ & $\begin{array}{c}1.60 \\
(0.05)\end{array}$ & $\begin{array}{c}1.50 \\
(0.04)\end{array}$ & 0.0418 \\
\hline
\end{tabular}

The comparison with the reference method from CASToR (with no blurring factors) proves that the complex arrangement of the detectors in J-PET has a significant impact on SRM. The intrinsic RM is shiftvariant, unlike the stationary Gaussian kernel available for image convolver in CASToR [18]. Our model has an additional advantage - the factors ignored in this work, such as positron range and non-collinearity, could be relatively easy included, either at the simulation stage or as separate kernels in Eq. (3).

The addition of the penalisation to TOF MLEM extends the number of adjustable parameters which can affect the outcome. A dedicated analysis of the impact of regularisation and/or the kernels in Eq. (3), is required, as well as how these factors work on other objects of the scan, such as whole-body phantoms. However, the exploratory analysis presented in this work showed that the proposed algorithm along with AMD-FMH may achieve image quality, comparable with much longer acquisition times. This potentially allows shorter time frames, low-dose imaging or radiotracers observed over longer periods.

\section{CONCLUSIONS}

We performed an exploratory study of the updated list-mode TOF MLEM image reconstruction algorithm that utilises realistically simulated SRM for the modular TB J-PET scanner. The application of the method to the 35-second scan of the NEMA IEC phantom, simulated in GATE, resulted in superior 
outcomes to the reference algorithm with no RM, in terms of RMSE and image quality. The proposed TOF MLEM is also flexible for the inclusion of additional physics and accounts for the unique regularities of the tomograph.

Among the penalisation methods included in the proposed algorithm, AMD-FMH proved to give the best results, close to the reconstructed images for the 500-second scan. However, further study is required in the future, including alternative objects of the scan.

Acknowledgements: This work was supported by the Foundation for Polish Science through MPD and TEAM 2017-4/39 programmes, the National Science Centre of Poland (grants No. 2016/21/B/ST2/01222, 2017/25/N/NZ1/oo861 and 2019/35/B/ST2/O3562), the Ministry of Science and Higher Education (grants No. 6673/IA/SP/2016, 715O/E-338/SPUB/2017/1 and $7150 / E-338 / M / 2017)$, the $E U$ and $M S H E$ through grant No. POIG.02.03.0o-161 Oo-013/o9 and the Austrian Science Fund (FWF-P26783).

\section{REFERENCES}

1. R. Ferrara, L. Mansi, "Paul Suetens (ed): Fundamentals of Medical Imaging (2nd edition)," Eur. J. Nucl. Med. Mol. Imaging, vol. 38, no. 2, 409, Feb. 2011. DOI: $10.1007 /$ soo259-010-1694-8

2. D. L. Bailey, D. W. Townsend, P. E. Valk, M. N. Maisey, Positron Emission Tomography: Basic Sciences, 1st ed., London, UK: Springer-Verlag, 2005.

Retrieved from: http://library.lol/main/62ECAB329922072DF347CA01 DC3AD401

Retrieved on: Jul. 14, 2021

3. S. Vandenberghe, P. Moskal, J. S. Karp, "State of the art in total body PET," EJNMMI Phys., vol. 7, 35, May. 2020

DOI: $10.1186 / \mathrm{s} 40658-020-00290-2$

PMid: 32451783

PMCid: PMC7248164

4. P. Moskal, E. Ł. Stepień, "Prospects and Clinical Perspectives of Total-Body PET Imaging Using Plastic Scintillators," PET Clin., vol. 15, no. 4, pp. 439 - 452, Oct. 2020.

DOI: 10.1016/j.cpet.2020.06.009 PMid: 32739047

5. R. D. Badawi et al., "First Human Imaging Studies with the EXPLORER Total-Body PET Scanner," J. Nucl. Med., vol. 60, no. 3, pp. 299 - 303, Mar. 2019.

DOI: 10.2967/jnumed.119.226498

PMid: 30733314

PMCid: PMC6424228

6. S. R. Cherry et al., "Total-Body PET: Maximizing Sensitivity to Create New Opportunities for Clinical Research and Patient Care," J. Nucl. Med., vol. 59, no. 1, pp. 3 - 12, Jan. 2018.

DOI: $10.2967 /$ jnumed.116.184028

PMid: 28935835 PMCid: PMC 5750522

7. P. Moskal et al., "Test of a single module of the J-PET scanner based on plastic scintillators," Nucl. Instrum. Methods Phys. Res. A, vol. 764, pp. 317 - 321, Nov. 2014. DOI: 10.1016/j.nima.2014.07.052

8. P. Moskal et al., "A novel method for the line-ofresponse and time-of-flight reconstruction in TOF-PET detectors based on a library of synchronized model signals," Nucl. Instrum. Methods Phys. Res. A, vol. 775, pp. 54 - 62, Mar. 2015 DOI: 10.1016/j.nima.2014.12.005

9. P. Moskal et al., "Time resolution of the plastic scintillator strips with matrix photomultiplier readout for J-PET tomograph,” Phys. Med. Biol., vol. 61, no. 5, pp. 2025 - 2047, Mar. 2016.

DOI: $10.1088 / 0031-9155 / 61 / 5 / 2025$

PMid: 26895187

10. S. Niedźwiecki et al., "J-PET: A New Technology for the Whole-body PET Imaging," Acta Phys. Pol. B, vol. 48 , no. 10, pp. 1567 - 1576, Oct. 2017. DOI: 10.5506/APhysPolB.48.1567

11. N. G. Sharma et al., "Hit-Time and Hit-Position Reconstruction in Strips of Plastic Scintillators Using Multithreshold Readouts," IEEE Trans. Radiat. Plasma Med. Sci., vol. 4, no. 5, pp. 528 - 537, Sep. 2020. DOI: 10.1109/TRPMS.2020.2990621

12. S. Sharma et al., "Estimating relationship between the time over threshold and energy loss by photons in plastic scintillators used in the J-PET scanner," EJNMMI Phys., vol. 7, 39, Jun. 2020.

DOI: $10.1186 / \mathrm{s} 40658-020-00306-\mathrm{x}$

PMid: 32504254

PMCid: PMC7275104

13. P. Moskal et al., "Simulating NEMA characteristics of the modular total-body J-PET scanner-an economic total-body PET from plastic scintillators," Phys. Med. Biol., vol. 66, no. 17, 175015, Sep. 2021.

DOI: $10.1088 / 1361-6560 / a c 16 b d$ PMid: 34289460

14. L. A. Shepp, Y. Vardi, "Maximum likelihood reconstruction for emission tomography," IEEE Trans. Med. Imaging, vol. 1, no. 2, pp. 113 - 122, Oct. 1982. DOI: 10.1109/TMI.1982.4307558 PMid: 18238264

15. A. J. Reader, H. Zaidi, "Advances in PET Image Reconstruction," PET Clin., vol. 2, no. 2, pp. 173 - 190, Apr. 2007.

DOI: 10.1016/j.cpet.2007.08.001 PMid: 27157872

16. S. Jan et al., "GATE: a simulation toolkit for PET and SPECT," Phys. Med. Biol., vol. 49, no. 19, pp. 4543 - 4561, Oct. 2004

DOI: $10.1088 / 0031-9155 / 49 / 19 / 007$

PMid: 15552416

PMCid: PMC 3267383

17. Performance Measurements of Positron Emission Tomographs (PETs), NEMA NU 2-2012, 2013. Retrieved from:

https://webstore.ansi.org/standards/nema/nemanu201 2-1451586\#PDF

Retrieved on: Jul. 14, 2021

18. T. Merlin et al., "CASToR: a generic data organization and processing code framework for multi-modal and multi-dimensional tomographic reconstruction," Phys. Med. Biol., vol. 63, no. 18, 185005, Sep. 2018.

DOI: $10.1088 / 1361-6560 /$ aadac1

PMid: 30113313

19. H. H. Barrett, T. White, L. C. Parra, "List-mode likelihood," J. Opt. Soc. Am. A, vol. 14, no. 11, pp. 2914 - 2923, Nov. 1997.

DOI: 10.1364/josaa.14.002914

PMid: 9379247

PMCid: PMC2969184

20. A. Strzelecki, "Image reconstruction and simulation of strip Positron Emission Tomography scanner using computational accelerators," Ph.D. dissertation, Polish Academy of Sciences, Institute of Fundamental Technological Research, Kraków, Poland, 2016. Retrieved from:

https://oldwww.ippt.pan.pl/ download/doktoraty/201 6strzelecki a doktorat.pdf

Retrieved on: Jul. 14, 2021

21. V. Westerwoudt, M. Conti, L. Eriksson, "Advantages of Improved Time Resolution for TOF PET at Very Low Statistics," IEEE Trans. Nucl. Sci., vol. 61, no. 1, pp. 126 - 133, Feb. 2014.

DOI: $10.1109 /$ TNS.2013.2287175

22. P. J. Green, "Bayesian reconstructions from emission tomography data using a modified EM algorithm," 
IEEE Trans. Med. Imaging, vol. 9, no. 1, pp. 84 - 93, Mar. 1990.

DOI: $10.1109 / 42.52985$

23. S. Alenius, U. Ruotsalainen, "Bayesian image reconstruction for emission tomography based on median root prior," Eur. J. Nucl. Med., vol. 24, no. 3, pp. 258 - 265, Mar. 1997.

DOI: $10.1007 / \mathrm{BF} 1728761$

PMid: 9143462

24. J. Nuyts, D. Beque, P. Dupont, L. Mortelmans, "A concave prior penalizing relative differences for maximum-a-posteriori reconstruction in emission tomography," IEEE Trans. Nucl. Sci., vol. 49, no. 1, pp. 56 - 60, Feb. 2002.

DOI: 10.1109/TNS.2002.998681

25. H. Ling, A. C. Bovik, "Smoothing low-SNR molecular images via anisotropic median-diffusion," IEEE Trans. Med. Imaging, vol. 21, no. 4, pp. 377 - 384, Apr. 2002. DOI: 10.1109/TMI.2002.1000261

PMid: 12022625
26. S. Alenius, U. Ruotsalainen, "Generalization of median root prior reconstruction," IEEE Trans. Med. Imaging, vol. 21, no. 11, pp. 1413 - 1420, Nov. 2002.

DOI: 10.1109/TMI.2002.806415

PMid: 12575878

27. J. Smyrski et al., "Measurement of gamma quantum interaction point in plastic scintillator with WLS strips," Nucl. Instrum. Methods Phys. Res. A, vol. 851, pp. 39 - 42, Apr. 2017.

DOI: $10.1016 /$ j.nima.2017.01.045

28. P. Kowalski et al., "Estimating the NEMA characteristics of the J-PET tomograph using the GATE package," Phys. Med. Biol., vol. 63, no. 16, 165008, Aug. 2018.

DOI: $10.1088 / 1361-6560 / a a d 29 b$ PMid: 29992906

29. S. Li, M. Wang, H. Hou, J. Yang, X. Wang, "Fast algorithm for calculating the radiological path in fanbeam CT image reconstruction," Optik, vol. 127, no. 5, pp. 2973 - 2977, Mar. 2016.

DOI: 10.1016/j.ijleo.2015.12.034 